\title{
COMPARACIÓN DE DESEMPEÑO EN LECTURA EVALUADA MEDIANTE UNA PRUEBA Y A TRAVÉS DE LA ESTIMACIÓN DEL MAESTRO
}

\author{
Lydia Pujol' y Eleonora Vivas² \\ Universidad Simón Bolivar
}

Se comparó el desempeño en lectura evaluada a través de una prueba estandarizada, con la evaluación realizada por los maestros, calculándose el grado de acucrdo entre ambas, en una muestra de 520 niños de escuela básica, en Caracas, Venezuela. El desempeño en Lectura fue definido como la habilidad para comprender y usar aquellas formas del lenguaje escrito requeridas por la sociedad y/o valoradas por el individuo. La prevalencia de dificultades en lectura obtenida mediante la prueba fue de $15 \% \mathrm{y}$ la estimada por los maestros fue del $40 \%$. Se evidenció un acuerdo moderado entre ambas medidas y una tendencia de los maestros a subestimar el logro en lectura en comparación con la prueba. Son discutidas las Implicaciones de los resultados.

Palabras clave: Lectura, niños, primaria, desempeño, maestro.

Comparison of reading achievement through a standarized test and the evaluation of the teacher

A comparison of reading achievement assessed by an standarized reading test with the prevalence estimated by the teachers was performed, by calculated agreement in a sample of 520 elementary school children in Caracas, Venezuela. Reading Achievement was defined as the ability to understand and use those forms of written language required by society and/or valued by the individuals. The prevalence of reading difficulties obtained from test scores was $15 \%$ and that obtained by the estimation made by teacher was $40 \%$. A moderate agreement between measured was obtained and a tendency of the teachers to understimate reading achievment was evidenced when compared with the results of the test. Implications of results are discussed.

Key words: Reading, children, elementary, school, achievement, teacher

1. Profesora asistente e investigadora del Departamento de Ciencia y Tecnologia del Comportamiento de la Universidad Simón Bolivar. Licenciada en Psicología de la Universidad Central de Venezuela y Magister en Psicología de la Universidad Simón Bolívar. Ha realizado investigaciones en comprensión de lectura, asesoramiento psicoeducativo y educación. superior. Correo electrónico: Ipujol@usb.ve.

2. Profesora del Departamento de Ciencia Y Tecnología del Comportamiento de la Universidad Simón Bolivar en Caracas, Vcnezuela. 

La planificación e implantación de programas a nivel comunitario con el fin de prevenir y/o remediar una condición, requiere como paso previo la medición del trastorno para obtener su frecuencia e identificar tempranamente las poblaciones en riesgo de presentar la problemática respectiva. Estos objetivos pueden lograrse mediante la investigación epidemiológica pues ella permite obtener la prevalencia del trastorno en poblaciones claramente definidas y determinar relaciones entre las variables involucradas, como por ejemplo el desempeño en lectura en la población escolar.

En el área de lectura, recientemente se ha focalizado la atención en identificar alternativas auténticas, confiables y eficientes para estimar el desempeño en lectura, el cual ha sido definido como "...la habilidad para comprender y usar aquellas formas del lenguaje escrito requeridas por la sociedad y/o valoradas por el individuo" (Elley, 1992). Este punto de vista conceptualiza el desempeño en lectura de manera funcional, ya que lo hace depender del contexto, haciendo énfasis en el grado de presión que ejerce la cultura para el aprendizaje de la lectura (Downing, 1973, en Elley, 1989), y como un continuo que implica diferentes niveles de desempeño (Chapman y Czerniewska 1978).

Con relación al método más idóneo para obtener la prevalencia del desempeño en lectura, no existe en la actualidad acuerdo entre los cspecialistas; unos se inclinan a favorecer la utilización de test estandarizados y otros hacia el empleo de la medición informal, es decir la estimación del desempeño efectuada por el maestro durante su labor diaria en el aula. Los que apoyan esta última práctica argumentan que son los expertos en el desarrollo de habilidades académicas las personas idóneas para apreciar el nivel de desempeño en lectura, debido a la consistencia observada en 
los índices de prevalencia de dificultades en esta habilidad obtenidos mediante este método, los cuales oscilan entre el $8 \%$ y el $15 \%$ en la población escolar (Bruiniks, Glaman y Clark, 1973).

Esta última postura es sostenida por Valencia y Pearson (1987), quienes opinan que la estimación del maestro es la mejor medida posible del logro en lectura, ya que ocurre cuando observan e interactuan con sus estudiantes al leer auténticos textos con propósitos genuinos y al intervenir proporcionando soporte y sugerencias cuando el estudiante parece tener dificultades, lo cual les permite tener una mejor apreciación de cómo se da el proceso en los alumnos de forma individual. Esta opinión es confirmada por Bell y Davey (1994), quienes afirman que las estrategias empleadas por el maestro para medir la lectura de manera informal (listas de chequeo, observaciones, inventarios de lectura) proporcionan una mejor opción que el test estandarizado, ya que están más centradas en evaluar formativamente aspectos relativos al proceso de lectura, a diferencia de la mayoría de las pruebas estandarizadas cuyo interés esta más centrado en el producto que en el proceso (Johnston, 1984). Sin embargo, Pumfrey y Kelly (en Pikulski 1978) advierten que la estimación del docente sólo es efectiva cuando se cuenta con maestros bien adiestrados en el registro e interpretación de los resultados obtenidos en la evaluación informal.

Por otra parte, en una investigación diseñada por Payne y Payne (1991) cuyos objetivos fueron comprobar la habilidad del docente para identificar alumnos con alto riesgo académico (bajo rendimiento), y verificar si sus apreciaciones estaban asociadas a factores que los educadores consideraban funcionalmente relacionados con el logro escolar, se contrastó la opinión del maestro con tres medidas de criterio (nivel socioeconómico, repitencia del último año escolar y los puntajes obtenidos en un test estandarizado de lectura). Se concluyó que los maestros son moderados estimadores del desempeño académico de sus alumnos, ya que se evidenciaron sesgos en sus opiniones basados en estereotipos $y$ creencias vinculadas con la raza y el nivel socioeconómico. Estos resultados están relacionados con los obtenidos por Espino y colaboradores 
(1987), en una muestra de 11.844 estudiantes de educación media, donde se encontró que la evaluación del rendimiento académico efectuado mediante notas (apreciación del docente) y pruebas académicas objetivas tenían poca coincidencia. Del análisis de los resultados se concluyó que posiblemente ambas medidas evaluaban distintos aspectos del rendimiento académico.

Otro aspecto a destacar, es la existencia de evidencia empírica que sugiere que las expectativas que posea el maestro sobre el rendimiento del alumno, influyen en el trato diferencial que le otorga al niño en el aula (Pumfrey, 1978; Good, 1987, Babad, 1995). Por ejemplo, si considera que el logro del alumno es bajo, tenderá a sentarlos más lejos de ellos en el aula, asignarles menos trabajos y otorgarles menos refuerzos y más críticas; siendo este comportamiento del maestro el posible responsable de la pobre ejecución escolar, pudiendo esta situación incidir en que se produzca la profecia autocumplida (Payne y Payne, 1991). Por otra parte, McAllister (1996) ha reportado evidencia sobre la tendencia de los docentes a otorgarse el crédito por el éxito de sus estudiantes y a no asumir la responsabilidad por los fracasos Estos aspectos conducen a formular la interrogante de cuán válidas son las estimaciones que sobre el rendimiento académico de sus pupilos formula el maestro.

Los defensores del empleo de pruebas estandarizadas sostienen que éstas permiten comparar de forma objetiva la ejecución de un estudiante en un momento en el tiempo, con la obtenida por la población de examinados en la cual se estandarizó la prueba (Farr, 1969). Apoyando esta posición, Elly (1989) sostiene que si el objetivo que se persigue es evaluar de forma sumativa y masiva el nivel de desempeño en lectura, es recomendable el uso de pruebas estandarizadas de lectura que empleen ítems de elección múltiple por su carácter práctico y cconómico. Un ejemplo de estos casos serían los estudios destinados a obtener la prevalencia de desempeño en lectura en una población determinada. En este sentido Farr (1969) recomienda el empleo de ambos tipos de medición de la lectura, mediante una prueba estandarizada y la evaluación informal del maestro, pues a pesar de los beneficios que ésta última aporta a la evaluación del proceso, tiene como problema que su validez depende 
como sugiere Morles (1994) del grado de conocimiento del docente relativo a las características individuales de sus estudiantes, del empleo de técnicas de evaluación apropiadas y de poseer el maestro el tiempo suficiente para realizarla. Además de estos aspectos, se requiere un profundo conocimiento sobre los procesos básicos de la lectura, habilidad para registrar errores y hacer juicios acerca de la ejecución lectora, cuyos criterios son completamente subjetivos.

En conclusión, las consideraciones anteriores parecen sugerir que la apreciación del maestro puede proporcionar una información completa del nivel de comprensión en lectura de un estudiante, siempre y cuando se trate de un docente bien preparado y experto en el área. Sin embargo existe el riesgo de que su evaluación obedezca a factores subjetivos, aspecto que podría controlarse más fácilmente con una prueba objetiva estandarizada.

Los estudios reportados en el ámbito internacional sobre la prevalencia de desempeño en lectura han empleado como método para medir la comprensión en lectura pruebas estandarizadas (Badian, 1984; Rutter, Tizard y Whitmore, 1970; Silva, Mac Gee y Williams, 1985). En Venezuela el único estudio epidemiológico sobre desempeño en lectura que ha sido reportado (Pujol, 1993; Pujol y Vivas, 1996) empleó ambos métodos para obtener la prevalencia (prueba estandarizada y apreciación del maestro); y se realizó con la base de datos del estudio internacional sobre desempeño en lectura efectuado por la I.E.A y la Universidad Pedagógica Libertador en Venezuela (1992), estando la presente investigación basada en parte de sus resultados.

Sobre la base de lo anteriormente expuesto, se ha considerado importante estimar el grado de acuerdo entre ambas medidas mediante la comparación de los índices de prevalencia obtenidos a través de una prueba de lectura bien construida, estandarizada y validada y la apreciación del maestro. Por otra parte, es relevante verificar si el grado de acuerdo entre ambas medidas varía en función de variables relativas a la preparación del docente como especialista en la enseñanza de la lectura. La 
constatación de estos aspectos proporcionará datos útiles de tomar en cuenta en el momento de planificar intervenciones tendientes a obtener indices de prevalencia del desempeño en lectura.

En consecuencia los objetivos en la presente investigación fueron: 1) Identificar mediante su comparación el grado de acuerdo entre la prevalencia calculada mediante una prueba estandarizada de lectura y la obtenida a través de la estimación del maestro, en una muestra representativa de la población escolar regular de la zona metropolitana de Caracas, cursante de $4^{\circ}$ grado de escuela Básica. 2) Identificar si el acuerdo entre la calificación obtenida a través de la prueba de lectura y la estimada por el maestro, esta relacionado con variables relativas a la preparación del maestro como facilitador del proceso de aprendizaje de la lectura.

\section{Métodología}

\section{Tipo de Estudio}

Estudio epidemiológico que describe la proporción de niños ubicados en los distintos niveles de desempeño en lectura (Nuy Pobre, Pobre, Promedio, Bueno, Muy Bueno). Igualmente, el estudio es correlacional comparativo, pues se busca la relación entre dos tipos de evaluación (la obtenida mediante la prueba vs. la apreciación del maestro).

\section{Patticipantes}

Se seleccionó 520 alumnos de un total de 98,891 que cursan el cuarto grado de escuela básica de primaria en Caracas. En el estudio no se consideraron escuelas de Fducación Especial ni remediales.

La muestra fue seleccionada mediante el azar sistemático. En la preparación del diseño muestral se empleó la técnica de la estratificación proporcional. Los estudiantes fueron seleccionados considerando los siguientes estratos de manera proporcional: (a) Ubicación del plantel 
donde cursan estudios (urbanos vs. rurales), (b) Dependencia del plantel (público vs. privado), (c) Distrito escolar del área metropolitana de Caracas.

La sección fue considerada como la unidad de muestreo y fueron tomadas como unidades intactas. El número promedio de alumnos por sección fue de veintisiete y el número de secciones contempladas fue de diecinueve.

Se estableció el número de secciones correspondientes a cada estrato y se seleccionó al azar el número de escuelas proporcionalmente planificadas para cada distrito y para cada estrato dentro de cada distrito. En las escuelas dónde existía más de una sección de $4^{\circ}$ grado, se seleccionó una aleatoriamente.

\section{Variables}

La variable que constituye el objeto principal del presente estudio epidemiológico es $d$ sempeño en lectura, la cual se dividió en cinco categorías las que fueron operacionalizadas del siguiente modo:

1. Desempeño muy Pobre: cuando el puntaje obtenido por el alumno en la prueba de lectura (I.E.A-U.P.E.L) se encuentre ubicado a menos de dos desviaciones estándar por debajo de la media de la distribución.

2. Desempeño Pobre: cuando el puntaje obtenido por el alumno en la prueba de lectura (I.E.A-U.P.E.L) se encuentre ubicado entre dos desviaciones estándar y menos de una desviación estándar por debajo de la media de la distribución.

3. Desempeño Promedio en Lectura: cuando el puntaje obtenido por el alumno en la prueba de lectura (I.E.A-U.P.E.L) se encuentre ubicado entre una desviación estándar por debajo de la media y una por encima de la media de la distribución.

4. Desempeño Bueno en Lectura: cuando el puntaje obtenido por el alumno en la prueba de lectura (I.E.A-U.P.E.L) se encuentre ubicado 
entre más de una desviación estándar por encima de la media de la distribución.

5. Desempeño Muy Bueno: cuando el puntaje obtenido por el alumno en la prueba de lectura (I.E.A-U.P.E.L) se encuentre ubicado a más de dos desviaciones estándar por encima de la media de la distribución.

Se consideraron otras variables relevantes para el problema en cuestión, como la preparación del maestro como facilitador del proceso de aprendizaje de la lectura. Entre éstas se seleccionaron el número de años cursados de educación primaria y secundaria, años de educación postsecundaria, años de prácticas docentes durante su formación como maestro, horas de clase dedicadas a la enseñanza de la lectura durante su formación docente, número de cursos de entrenamiento en lectura para maestros en servicio en los últimos tres años, número total de años de dedicación a la docencia y frecuencia de los diferentes tipos de lectura que realiza el maestro.

\section{Instrumentos}

Prueba de Desempeño en Lectura. Para medir el nivel de desempeño en lectura se empleó la prueba elaborada por la Asociación Internacional Para la Evaluación del Logro Educativo (I.E.A.) y la Universidad Pedagógica Libertador (U.P.E.L.), para el Estudio Internacional de Desempeño en Lectura (I.E.A, 1992). La prueba consta de 51 ítemes generados de 18 textos (5 narrativos, 5 expositivos y 7 documentales). El puntaje máximo que obtiene un sujeto es de 51 puntos (un punto por cada ítem contestado correctamente). En Venezuela la prueba fue sometida a una validación tanto idiomática como de contenido y se obtuvo un coeficiente de consistencia interna de 0.95 (Alfa de Crombach). Se hizo hincapié en la validez ecológica, es decir que la información contenida en la prueba estuviera estrechamente relacionada con actividades de lectura de la vida cotidiana, que tuviesen sentido para los niños venezolanos.

Para obtener la estimación del nivel de desempeño en lectura efectuada por los maestros, se diseñó un instructivo dirigido al docente para 
facilitarles esta labor. Alli se les explicaba como clasificar a sus alumnos de acuerdo a las siguientes categorías descriptivas:

1. Lectores Muy Pobres: Estos demuestran consistentemente poca comprensión sobre lo que leen. Sus respuestas sobre la lectura son muy generales y pocas veces van más allá de la interpretación. Frecuentemente proporcionan información errada en respuesta a una pregunta.

2. Lectores Pobres: Pueden generalizar basados en una sola dimensión y frecuentemente omiten información relevante dentro del texto.

3. Lectores Promedio: Tienden a considerar varias opciones y pueden desarrollar varias generalizaciones basándose en la combinación de informaciones dadas por los materiales, pero con frecuencia no toman en cuenta todas las alternativas dadas por el texto.

4. Iectores Buenos: Tienden a usar todas las informaciones del texto, discriminando encre información relevante y no relevante. Pueden llegar a generalizaciones y se dan cuenta de variedad de posibilidades; además elaboran conclusiones propias partiendo de su experiencia personal.

5. Lectores Muy Butizos: forman generalizaciones combinando información del texto y experiencia propia, tomando en cuenta interpretaciones alternativas con mayor eficiencia que los buenos lectores. Pueden valorar sus propias generalizaciones y aplicarla a nuevas situaciones Y nuevos contextos.

6. No Se: En algunas situaciones pudiera ocurrir que el docente no esté familiarizado con los niveles de lectura de sus estudiantes; por ejemplo, en el caso de un estudiante recién incorporado a la sección. En estos casos se debía aplicar esta categoría.

Cuestionario del Docente. Fue diseñado para el estudio internacional de la I.E.A (1992). Tiene como finalidad obtener información sobre el maestro que conduce el aprendizaje; para el presente estudio se tomó la referente a preparación académica y hábitos de lectura. 


\section{Procedimiento}

Se llevó a cabo en dos fases, en primer término se efectuó un taller de entrenamiento dirigido a estudiantes universitarios que se desempeñaron como aplicadores de la prueba. Posteriormente se aplicó la prueba en dos sesiones, con el fin de evitar dificultades en su completación debidas a fatiga o desinterés. Durante la primera sesión se le entregó al maestro el instructivo dónde se le explicaba al docente las cinco categorías mediante las cuales debía estimar el desempeño en lectura de sus estudiantes, conjuntamente con el Cuestionario del Docente indicándoles que debian entregarlos en la siguiente sesión.

\section{Resultados y discusión}

Con el objeto de comparar el grado de acuerdo entre la prevalencia de desempeño en lectura obtenida a través de la prueba con la estimada mediante la apreciación del maestro, se elaboró una matriz de contingencia (Cuadro 1) y se calculó el coeficiente Kappa (Cohen, 1960) para estimar el grado de acuerdo entre escalas nominales. La fórmula para su cálculo se define como la proporción de acuerdos observados (Po) corregida explícitamente por la de acuerdos aleatorios (Pc).

$$
\begin{aligned}
& \mathrm{P}_{0}=\frac{\text { SumaValoresDiagonal }}{\text { Total }}=\frac{179}{520}=0.3442307 \\
& \mathrm{P}_{c}=\sum_{1}^{K}\left[P_{1} \times P_{2}\right]=0.3003595 \\
& \text { Kappa }(k)=\frac{\left(\mathrm{P}_{0}-\mathrm{P}_{c}\right)}{\left(1-\mathrm{P}_{c}\right)}=0.0627008
\end{aligned}
$$

En el Cuadro 1 se observan los acuerdos y desacuerdos según los datos correspondientes se coloquen en la diagonal principal o fuera de ella. Se evidencia que en las categorías de desempeño en lectura calculadas con la prueba, la frecuencia mayor de alumnos tiende a agruparse hacia 
la línca media, ubicándose el $69 \%$ de la muestra en la categoría correspondiente a desempeño en lectura promedio, siguiendo una distribución similar a la curva normal. Sin embargo, los puntajes obtenidos mediante la apreciación del maestro tienden a dispersarse principalmente entre las categrorías 2,3 y 4 . Se obscrvó que el mayor número de acuerdos se produce en la categoría de desempeño en lectura promedio. En general se evidencia una tendencia de los maestros a estimar en niveles más bajos el desempeño de sus alumnos en comparación con los resultados obtenidos en la prueba, ubicando en las categorias 1 y 2 indicativa de dificultades en el desempeño en lectura (Desempeño Pobre y Muy Pobre) el $40 \%$ de la muestra, mientras que la prueba agrupa el $15 \%$ en los mismos niveles.

\section{Cuadro 1}

Nuimero de Acuerdos y Desacuerdos entre las Calificaciones de la Prueba y la Estimación del Maestro por Categoría de Desempeño en Lectura

\begin{tabular}{|c|c|c|c|c|c|c|c|}
\hline $\begin{array}{l}\text { Estimación } \\
\text { maestro }\end{array}$ & $\begin{array}{l}\text { Cat } \\
1\end{array}$ & $\begin{array}{c}\text { goría } \\
2\end{array}$ & $\begin{array}{l}\text { según } \\
3\end{array}$ & $\begin{array}{l}\text { la pru } \\
4 \quad 5\end{array}$ & eba & Total & Proporción \\
\hline 1 & 1 & 18 & 57 & 2 & 0 & 78 & 0.15 \\
\hline 2 & 6 & 27 & 84 & 13 & 0 & 130 & 0.25 \\
\hline 3 & 2 & 17 & 126 & 30 & 2 & 177 & 0.34 \\
\hline 4 & 0 & 5 & 72 & 23 & 4 & 104 & 0.2 \\
\hline 5 & 0 & 2 & 20 & 7 & 2 & 31 & 0.06 \\
\hline Total & 9 & 69 & 359 & 75 & 8 & 520 & \\
\hline Proporción & 0.017 & 0.133 & 0.69 & 0.144 & 0.015 & & \\
\hline
\end{tabular}


Estos resultados nos inducen a pensar que los maestros poseen mayor facilidad para estimar un nivel medio de lectura, presentando mayores dificultades para apreciar los niveles más bajos de desempeño en lectura, los cuales requieren de mayor experticia en el área para su estimación precisa.

El Coeficiente de Kappa (0.062) sugiere un grado de acuerdo no muy elevado entre ambas apreciaciones de la prevalencia. Sin embargo al analizar los resultados de este coeficiente hay que tomar en cuenta que la relación que mide esta basada en el número de acuerdos por categoría, la cual es una relación muy puntual y exacta entre dos condiciones que no debe confundirse con la mera covariación entre dos variables. Estos resultados sugieren que si en lugar de cinco categorías se hubiesen empleado tres, el acuerdo entre los cálculos de la prevalencia posiblemente sería más alto, debido a que el maestro al tener menos opciones dispersarian menos sus estimaciones.

Por consiguiente, al comparar la prevalencia de desempeño en lectura calculada mediante la prueba estandarizada con la estimada por el maestro, se puede concluir que el acuerdo entre ambas fue bajo. Este resultado podría tener varias explicaciones, una sería la sugerida por Espino y Colaboradores (1987) de que ambas medidas evalúan aspectos diferentes del desempeño en lectura. Es decir, con la prueba se obtiene una estimación de un momento en el tiempo, un producto puntual relativo a los aspectos de la habilidad en lectura contemplados en el test; a diferencia de la estimación del desempeño en lectura efectuada por un maestro, adecuadamente capacitado y con el tiempo suficiente para conocer a sus alumnos, a través de la cual pueden observarse detalles del proceso de adquisición de la lectura que no son posibles de determinar a través de una prueba masiva, con ítems de elección múltiple o completación (Bell y Sammons, 1994; Johnston, 1984; Valencia y Pearson, 1987). Otra razón que pudiera explicar el moderado acuerdo entre ambas medidas, es que la apreciación del docente en ocasiones puede estar sesgada debido a sus actitudes y creencias, como lo señalan Payne y Payne (1991), Babad (1995) y McAllister (1996). 
A continuación se analizará el grado de acuerdo y la preparación del maestro. Con el fin de identificar si el grado de acuerdo entre la calificación obtenida por un alumno en la prueba de desempeño en lectura y la estimada por su maestro, estaba relacionado con variables relativas a la preparación del docente como facilitador del proceso de aprendizaje de la lectura, se calculó el porcentaje de acuerdo general entre ambas medidas en todas las categorías (Cuadro 2), el porcentaje de acuerdos en las categorias 1 y 2 (Muy pobre y Pobre) indicativas de la presencia de dificultades en el desempeño en lectura (Cuadro 3) siendo estas categorías las que presentan la media de porcentaje de acuerdos más baja (25\%), el porcentaje de acuerdo en la categoria 3 (Promedio) (Cuadro 3) y porcentaje de acuerdo en las categorias 4 y 5 (Bueno y Muy Bueno) (Cuadro 3). Estas cuatro variables se correlacionaron con las siguientes: número de años de educación escolar del docente, años de prácticas docentes durante su formación como maestro, años de educación universitaria, boras de clase dedicadas a la enseñanza de la lectura en su formación como docente, numero total de años dedicados a la docencia, numero de cursos de entrenamiento sobre lectura para maestros en servicio en los uiltimos tres años, frecuencia de lectura de diversos materiales (articulos de enseñanza, articulos sobre enseñanza de la lectura, libros de: listoria, arte, ciencias, novelas, poemas y juegos infantiles). También se consideró importante correlacionar la variable Porcentaje de Acuerdo General con el número de estudiantes en el aula de cada maestro.

En el análisis de la matriz de correlación se observó que la variable Porcentaje de Acuerdo General presentó las correlaciones más altas, positivas y significativas con las siguientes variables: Años de Educación Universitaria $(r=0.46, p=.05)$ y Años de Práctica Docente en su Formación como Maestro $(r=0.45, p=.05)$. Con relación a la variable Porcentaje de Acuerdos en las Categorias Muy Pobre y Pobre, se evidenció que correlacionó posịtiva y significativa-mente con Años de dedicación a la Docencia $(r=0.55, p=.01)$. El Porcentaje de Acuerdo en la Categoría Promedio correlacionó positiva y significativamente con Lectura de Libros de Poemas por parte del Maestro $(r=0.53, p=.05)$, Lectura de Artículos sobre Enseñanza por parte del Maestro $(r=0.40, p=.05)$ y Años de Prácticas Docentes en su Formación como Maestro $(r=0.49, p=.05)$. La variable Porcentaje de Acuerdo en las Categorias Bueno $y$ 
Desempeño en lectura

\section{Cuadro 2}

Número y Porcentaje de Acuerdo General de cada Maestro en Relación a la Prueba de Lectura en todas las Categorias

\begin{tabular}{|rccc|}
\hline Maestro & $\begin{array}{c}\text { Estudiantes } \\
(n)\end{array}$ & $\begin{array}{c}\text { Acuerdos } \\
(n)\end{array}$ & $\begin{array}{c}\text { Acuerdos } \\
(\%)\end{array}$ \\
\hline 1 & 33 & 1 & 3.03 \\
2 & 32 & 11 & 34.38 \\
3 & 35 & 9 & 25.71 \\
4 & 34 & 12 & 35.29 \\
5 & 35 & 22 & 62.86 \\
6 & 29 & 7 & 24.14 \\
7 & 30 & 12 & 40.00 \\
8 & 32 & 8 & 25.00 \\
9 & 25 & 8 & 32.00 \\
10 & 28 & 9 & 32.14 \\
11 & 15 & 5 & 40.00 \\
12 & 28 & 11 & 17.86 \\
13 & 35 & 9 & 31.43 \\
14 & 11 & 9 & 81.82 \\
15 & 20 & 24 & 45.00 \\
16 & 37 & 4 & 64.86 \\
17 & 16 & 7 & 25.00 \\
18 & 23 & 5 & 30.43 \\
19 & 22 & 9 & 22.73 \\
\hline $\bar{X}$ & 27 & $55 \%$ \\
\hline
\end{tabular}

Muy Bueno correlacionó positiva y significativamente con Años de Prácticas Docentes en su Formación como Maestro $(r=.52, p=.05)$.

Estos resultados sugieren que a medida que el maestro posee más años de estudios universitarios y ha tenido más horas de prácticas docentes en su formación, aumenta el número de veces que su apreciación sobre el desempeño en lectura de sus alumnos coincide con el indicado en la prueba estandarizada en todas las categorías. Sin cmbargo, en las categorias Pobre y Muy Pobre, indicativas de la presencia de dificultades en el desempeño en lectura, la relación encontrada parece indicar que los años 


\section{Cuadro 3}

Número de Estudiantes (E) Ubicados por la Prueba en las Categorías. Nuimero y Porcentaje de Acuerdos (A) de cada Maestro ẹn Relación a la Prueba para cada Categoria

\begin{tabular}{|c|c|c|c|c|c|c|c|c|c|}
\hline \multicolumn{10}{|c|}{ Categoria } \\
\hline \multicolumn{4}{|c|}{$\begin{array}{c}\text { Muy Pobre } \\
\text { y Pobre }\end{array}$} & \multicolumn{3}{|c|}{ Promedio } & \multicolumn{3}{|c|}{$\begin{array}{l}\text { Bueno y } \\
\text { Muy Bueno }\end{array}$} \\
\hline Maestro & $\begin{array}{l}\mathrm{E} \\
(n)\end{array}$ & $\begin{array}{l}\mathrm{A} \\
(n)\end{array}$ & $\begin{array}{c}\text { A } \\
(\%)\end{array}$ & $\begin{array}{l}\mathrm{E} \\
(n)\end{array}$ & $\begin{array}{l}\text { A } \\
(n)\end{array}$ & $\begin{array}{c}\text { A } \\
(\%)\end{array}$ & $\begin{array}{l}\mathrm{E} \\
(n)\end{array}$ & $\begin{array}{l}\text { A } \\
(n)\end{array}$ & $\begin{array}{c}A \\
(\%)\end{array}$ \\
\hline 1 & 2 & 0 & 0 & 24 & 1 & 4.2 & 7 & 0 & 0 \\
\hline 2 & 2 & 0 & 0 & 23 & 7 & 30.5 & 7 & 4 & 57.1 \\
\hline 3 & 8 & 3 & 37.5 & 25 & 4 & 16.0 & 2 & 2 & 100.0 \\
\hline 4 & 2 & 0 & 0 & 15 & 6 & 40.0 & 17 & 6 & 35.3 \\
\hline 5 & 9 & 7 & 77.8 & 24 & 13 & 54.2 & 2 & 2 & 100.0 \\
\hline 6 & 4 & 2 & 50.0 & 23 & 4 & 17.4 & 2 & 1 & 50.0 \\
\hline 7 & 3 & 1 & 33.3 & 18 & 10 & 55.6 & 9 & 1 & 11.0 \\
\hline 8 & 9 & 1 & 11.1 & 22 & 7 & 31.8 & 1 & 0 & 0 \\
\hline 9 & 1 & 0 & 0 & 20 & 7 & 35.0 & 4 & 1 & 25.0 \\
\hline 10 & 4 & 1 & 25.0 & 23 & 8 & 34.8 & 1 & 0 & 0 \\
\hline 11 & 0 & 0 & 0 & 8 & 4 & 50.0 & 7 & 2 & 28.0 \\
\hline 12 & 6 & 3 & 30.0 & 20 & 2 & 10.0 & 2 & 0 & 0 \\
\hline 13 & 3 & 1 & 33.3 & 22 & 8 & 36.4 & 10 & 2 & 20.1 \\
\hline 14 & 1 & 0 & 0 & 8 & 7 & 87.5 & 2 & 2 & 100.0 \\
\hline 15 & 4 & 3 & 75.0 & 13 & 6 & 46.2 & 3 & 0 & 0 \\
\hline 16 & 8 & 3 & 37.5 & 26 & 20 & 76.9 & 3 & 1 & 33.3 \\
\hline 17 & 3 & 0 & 0 & 12 & 3 & 25.0 & 1 & 1 & 100.0 \\
\hline 18 & 7 & 3 & 42.8 & 15 & 4 & 26.7 & 1 & 0 & 0 \\
\hline 19 & 2 & 0 & 0 & 18 & 5 & 27.8 & 2 & 0 & 0 \\
\hline $\bar{x}$ & 4 & 1 & $25 \%$ & 19 & 7 & $37 \%$ & 4 & 1 & $35 \%$ \\
\hline
\end{tabular}


de experiencia del maestro en la docencia tienen gran peso para poder distinguir a los pobres lectores de los que no lo son. Los datos reflejan que esta tendencia se incrementa a partir de los 10 años dedicados a la docencia. El incremento del grado en que el maestro coincide con la prueba en la estimación de la Categoría Promedio, esta relacionada con la frecuencia y tipo de lectura que éste realiza, específicamente de artículos de enseñanza y de poemas, además del mayor número de años de prácticas docentes. Con relación a este punto se evidenció que el máximo número de acuerdos se establecía en aquellos maestros que habian tenido 4 ó 5 años de prácticas. En cuanto a la estimación acertada de las Categorías de Desempeño Bueno y Muy Bueno también esta vinculada con el mayor número de años de práctica docente, lo que parece contribuir a que el maestro adquiera mayor experticia en precisar el nivel de desempeño en lectura que presentan sus alumnos.

Vale señalar que no hay una variable única relativa a la preparación del maestro que se relacione con la coincidencia de calificaciones para el lector. Las relaciones sugieren variables diferentes de acuerdo a si el lector es pobre, promedio o bueno. Sólo un mayor número de años de estudio estaría relacionado con acuerdos en todas las categorías. Otra variable que parece importante para el grado de acuerdo es el número de años de prácticas docentes. De esta manera podemos observar que cada variable dentro de la formación del maestro sería importante para ayudarle a éste a discriminar entre diferentes tipos de lectores.

Con el fin de determinar dentro del conjunto de variables estudiadas relacionadas con la preparación del maestro, cual de ellas son las mejores predictoras del grado de acuerdo (tanto general como en cada categoría) entre la estimación del desempeño en lectura efectuada por el maestro y la calificación obtenida en la prueba de lectura, se realizó un análisis de regresión múltiple por etapas (stepwise). Las variables que efectuaron un mayor aporte en la predicción del grado de acuerdo general (ver Cuadro 4) fueron: Años de Prácticas Docentes en su Formación, donde se observa que hay una relación positiva y significativa $(F(1,11)=6.06 ; p=.031)$ con un porcentaje de variación explicado de 35,48\%; Frecuencia de Lectura de Libros sobre 
Ciencias, presentando una relación negativa y significativa $(F(2,10)=9.78$; $p=.0004$ ) con un porcentaje de variación explicado de $66.19 \%$. Lectura de Articulos sobre Enseñanza evidencia una relación positiva y significativa $F(3$, 9) $=15,04 ; p=.0007$ ) con un porcentaje de variación explicado de $83,37 \%$; Lectura de Libros sobre Historia mostró una relación negativa y significativa $(F(4,8)=21,89 ; p=.0002)$ con un porcentaje de variación explicado de $91,63 \%$. Estos resultados indican que el incremento del número de años de prácticas docentes, la mayor frecuencia de lectura de artículos sobre enseñanza y la menor frecuencia de lectura de libros sobre ciencias e historia, están asociados a un mayor porcentaje general de acuerdos entre la calificación obtenida en la prueba de lectura y la estimación del desempeño efectuada por el maestro.

Del análisis de estos datos se puede inferir que un maestro que cuenta con más años de pasantías en su formación como docente (de cuatro a cinco años) tiene mavor oportunidad de obtener experiencia bajo la supervisión de expertos sobre el proceso de enseñanza aprendizaje en general y de la lectura en particular; lo cual favorecerá el que aprecie con más acierto los difcrentes niveles de desempeño de los alumnos.

En cuanto a la relación negativa observada con las variables Lectura de Libros sobre Cienia e Historia, se podrian elaborar varias inferencia: e hipótesis explicativas a ser comprobadas en futuros estudios, como scría el caso de suponer que los maestros que más frecuentemente leen sobre estos temas sean los docentes graduados en Educación Básica con mención en Ciencias o Historia, lo cual podría sugerir que estos profesionales no tienden a discriminar acertadamente el nivel de desempeño en lectura de sus alumnos, quizá porque su interés esté más dirigido hacia otras áreas diferentes al desempeño en lectura.

Las variables que aportaron mayor ganancia en la predicción del grado de Acuerdo en las Categorias Muy Pobre y Pobre fueron (ver Cuadro 5): Años de Dedicación a la Docencia, donde se observa una relación positiva y significativa $(F(1,11)=7,45 ; p=.019)$ con un porcentaje de variación explicada del 40,40\%, y Lectura de Articulos sobre Enseñanza de la Lectura, 
Desempeño en lectura

\section{Cuadro 4}

Análisis de Regresión por Etapas para el Porcentaje de Acuerdo General entre la Prueba de Lectura y la Estimación del Maestro

\begin{tabular}{|lllccc|}
\hline & \multicolumn{1}{c}{ Variable } & B & EEB & $\beta$ & $R^{2}$ \\
\hline Paso 1 & $\begin{array}{l}\text { Prácticas Docentes } \\
\text { Paso 2 }\end{array}$ & 10.95 & 1.53 & 0.77 & 0.35 \\
& $\begin{array}{l}\text { Lectura Libros } \\
\text { sobre Ciencia } \\
* \rho \leq .004\end{array}$ & -10.16 & 1.84 & -5.53 & 0.66 \\
Paso 3 & $\begin{array}{l}\text { Lectura Artículos } \\
\text { sobre Enseñanza } \\
* \rho \leq .0007\end{array}$ & 10.51 & 2.16 & 0.70 & 0.83 \\
Paso 4 & $\begin{array}{l}\text { Lectura Libros } \\
\text { sobre Historia } \\
* \rho \leq .0002\end{array}$ & -6.97 & 2.48 & -0.42 & 0.91 \\
& & & & & \\
\hline
\end{tabular}

evidenciando una relación negativa y significativa $(F(2,10)=7,88 ; p=.008)$ con un porcentaje de variación explicado de $61,19 \%$.

No luce sorprendente que la mejor variable predictora del grado de acuerdo en la categoría Muy Pobre y Pobre sea el número de años dedicado a la docencia, esta relación implica que a más años de labor docente el maestro se hace más experto en detectar a los estudiantes con dificultades en la lectura. Sin embargo la segunda variable predictora de estas categorias luce contradictoria ya que sugiere que una menor frecuencia de lectura de artículos sobre enseñanza de la lectura esta asociado a un mayor grado de acuerdo en la estimación de los pobres lectores; esta relación requiere de una investigación más profunda a fin de dilucidarla.

En cuanto a la variable Porcentaje de Acuerdo en la Categoria Promedio (ver Cuadro 6) se encontró que sus mejores predictores son: Años de Prácticas Docentes dónde se observa una relación positiva y significativa $(F(1,11)=6.13$; $p=.030$ ), con un porcentaje explicado del 35,78\%; Lectura de Libros sobre 


\section{Cuadro 5}

Análisis de Regresión por Etapas para el Porcentaje de Acuerdos entre la Prueba de Lectura y la Estimación del Maestro en las Categorias Muy Pobre y Pobre de Desempeño en Lectura

\begin{tabular}{|c|c|c|c|c|c|}
\hline & Variable & $\mathrm{B}$ & EEB & $\beta$ & $R^{2}$ \\
\hline Paso 1 & $\begin{array}{l}\text { Años de Dedicación } \\
\text { a la Docencia } \\
* \rho \leq 0.0196\end{array}$ & 4.56 & 1.20 & 0.79 & 0.40 \\
\hline Paso 2 & $\begin{array}{l}\text { Lectura Artículos sobre } \\
\text { Enseñanza de la Lectura } \\
* \rho \leq 0.0088\end{array}$ & -9.54 & 4.12 & -0.48 & 0.61 \\
\hline
\end{tabular}

\section{Cuadro 6}

Análisis de Regresión por Etapas para el Porcentaje de Acuerdos entre la Prueba de Lectura y la Estimación del Maestro en la Categoria Promedio de Desempeño en Lectura

\begin{tabular}{|lllclc|}
\hline & \multicolumn{1}{c}{ Vuriable } & B & EEB & $\beta$ & $R^{2}$ \\
\hline Paso 1 & $\begin{array}{l}\text { Prácticas Docentes } \\
* \rho \leq 0.030\end{array}$ & 11.85 & 1.60 & 0.76 & 0.35 \\
Paso 2 & $\begin{array}{l}\text { Lectura Libros } \\
\text { sobre Ciencia } \\
* \rho \leq 0.014\end{array}$ & -9.47 & 1.92 & -0.50 & 0.57 \\
Paso 3 & $\begin{array}{l}\text { I.ectura Articulos } \\
\text { sobre Enseñanza } \\
* \rho \leq 0.001\end{array}$ & 13.48 & 2.26 & 0.82 & 0.81 \\
Paso 4 & $\begin{array}{l}\text { Lectura Libros } \\
\text { sobre Historia } \\
* \rho \leq 0.002\end{array}$ & -8.88 & 2.60 & -0.48 & 0.92 \\
& & & & & \\
\hline
\end{tabular}


Ciencia presentando una relación negativa y significativa $(F(2,10)=6.71$; $p=.014)$ con un porcentaje de variación explicado de 57,30; Lectura de Articulos de Enseñanza evidenciando una relación positiva y significativa $F(3$, 9) $=12,92 ; p=.001$ ) con un porcentaje de variación explicado de $81,16 \%$; Lectura de Libros Sobre Historia donde se observa una relación negativa y significativa $(F(4,8)=24,15 ; p=.0002)$ con un porcentaje de variación de $92,35 \%$. Estas cuatro variables son las mismas que predicen el Porcentaje de Acuerdo General, siendo lógico este resultado ya que es en la categoría Promedio dónde se presentan el mayor número de acuerdos, como se ilustra en la matriz para el cálculo de coeficiente Kappa (Cuadro 1) y el mayor promedio de porcentaje de acuerdos (37\%) (Cuadro 3).

La mejor variable predictora para Porcentaje de Acuerdo en la Categoría Bueno y Muy Bueno (Cuadro 7) es Número de Horas de Prácticas Docentes observándose una relación positiva y significativa $(F(1,11)=15,56 ; p=.0023)$ con un porcentaje de variación explicando $58,58 \%$.

\section{Cuadro 7}

Análisis de Regresión por Etapas para el Porcentaje de Acuerdos entre la Prueba de Lectura y la Estimación del Maestro en las Categorias Bueno y Muy Bueno de Desempeño en Lectura

\begin{tabular}{|lllccc|}
\hline & Variable & B & EEB & $\beta$ & $R^{2}$ \\
\hline Paso 1 & $\begin{array}{l}\text { Prácticas Docentes } \\
* \rho \leq 0.002\end{array}$ & 24.12 & 6.12 & 0.77 & 0.58 \\
& & & & & \\
\hline
\end{tabular}




\section{Conclusiones y recomendaciones}

Sobre la base de los resultados obtenidos al comparar el grado de acuerdo entre la prevalencia de desempeño en lectura calculada mediante una prueba estandarizada con la estimada por el maestro, se puede concluir que:

- El acuerdo entre ambas no fue muy elevado según se evidencia por el calculo del coeficiente Kappa. Este resultado puede obedecer a diferentes razones. Por un lado, es posible que los maestros están evaluando aspectos diferentes a los que considera la prueba de desempeño en lectura y por ende al realizar las comparaciones, éstas evidencian una baja coincidencia. Pero, por otra parte pudiera estar relacionado con una preparación insuficiente de los docentes para facilitar y evaluar el proceso de lectura en sus alumnos. Otra explicación del bajo acuerdo en el cálculo de ambas prevalencias, es que las evaluaciones de los docentes pudieran presentar sesgos basados en estereotipos y creencias como la raza, nivel socioeconómico y expectativas de logro en relación al alumno (Pumfrey 1978, Good 1987, Payne y Payne, 1991).

- Los resultados indican que los mejores predictores de acuerdo entre las evaluaciones de los maestros y una prueba estandarizada cuando se trata de niños de rendimiento promedio y alto, son un elevado número de prácticas docentes y la lectura de artículos sobre enseñanza. Sin embargo, la variable que predice mayor grado de acuerdo cuando de niños de bajo o pobre rendimiento en lectura se trata, son los años de dedicación a la docencia que tiene el maestro. Esto es un indicador de que se necesitan maestros con gran experiencia para poder discriminar cuando un niño tiene deficiencias en lectura. Estos datos corroboran lo expresado por Pumfrey (1978) de que la estimación del docente es efectiva si cuentan con la adecuada experiencia y preparación.

- En general, los resultados obtenidos en este estudio parecen apoyar la sugerencia de Farr (1969) de emplear ambos tipos de medida del 
desempeño en lectura (prueba estandarizada y estimación del maestro). Mediante el uso de la prueba se pueden contrarrestar los sesgos debidos a la subjetividad del maestro, además de permitir la comparación de la calificación obtenida por un alumno con la de sus pares en un momento determinado del proceso. Este aspecto es de suma importancia cuando se trata de llevar a cabo estudios epidemiológicos para obtener la prevalencia del desempeño en lectura. Por otra parte, el contar con la estimación del desempeño en lectura efectuada por maestros con vasta experiencia y bien preparados en el área, permite apreciar en forma certera el proceso de aprendizaje de la lectura de cada estudiante y en consecuencia una mejor comprensión de su nivel de desempeño para ese momento.

Estos resultados tienen implicaciones importantes relacionadas con las políticas educativas de formación y actualización del personal docente destinado a la educación básica, ya que reflejan el desconocimiento que presenta un gran número de docentes del nivel de desempeño en lectura de sus pupilos; evidenciando la necesidad de contar con maestros mejor preparados en la facilitación y evaluación del proceso de lectura.

\section{Referencias}

Babad, E. (1995). The teacher's pet phenomenon, student's perceptions of teacher's differential behavior, and student's morale. Joumal of Educational Psychology, 87 (3), 361-364.

Badian, N. (1984). Reading disability in an epidemiological contex incidence and enviromental correlates. Joumal of Learning Disabilities, 17 (3), 129 136.

Bell, R. y Davey, B. (1994), Assessing students'skills in using textbook awareness and performance profile (tapp). Joumal of Reading. 37 (4), 280-286.

Bruiniks, R; Glaman, \& Clark,Ch.. (1973) Issues in determining prevalence of reading retardation. The Reading Teacher. 27 (2), 177-185. 
Chapman, J; Czerniewska, P. (1978). Looking at literacy. En J. Chapman \& P. Czerniewska (Eds.). Reading from process to practice (pp. 3-5). Londres: Henley Routledge \& Kegan Paul in Association with the Open University.

Elley, W. (1989). Reviem of researcb on reading literacy. Manuscrito no publicado, The International Association for the Evaluation of Educational Achievement.

Elley, W. (1992). How in the world do students read? I.E.A. Study of Reading Literacy. Hamburgo: The International Association for the Evaluation of Educational Achievement.

Espino, M, Marrero, H; Díaz, M; Buela, G; y Echevarria, M. (1987, septiembre). ¿Uno o dos rendimientos académicos?. Evaluación del rendimiento académico a través de notas escolares. Memorias del II Congreso de Evaluación Psicologica. Madrid, pp. 22-27.

Farr, R. (1969). Reading: what can be measured? Delaware: International Reading Asociation

Good, T. (1987). Two decades of research on teacher's expectation: finding and future directions. Joumal of Teacher Educations. 38 (4), 3244.

Johnston, P. (1978) Assessment in reading. En P.D. Pearsons (Ed.), Handbook of reading research. Nueva York: Logman.

McAllister, H. (1996). Self serving bias in the classroom: who knows it? Joumal Educational Psychology.

Morles, A. (1994). Fl conocimiento de los docentes sobre la capacidad para leer de sus estudiantes. Educación y Ciencias Humanas. 2 (3), 113126.

Payne, B. y Payne, D. (1991). The ability of teachers to identify academically at risk elementary students. Journal of Research in Childbood Education. 5 (2), 116-126.

Pikulski, J.(1978). A critical review: informal reading inventories. En J. Chapman \& P. Czerniewska (Eds.). Reading from process to practice (pp. 3-5). Londres: Henley Routledge \& Kegan Paul in Association with the Open Lniversity. 
Pujol, L (1993). Estudio epidemiologico sobre desempeño en lectura y su relación con otras variables. Tesis de maestría no publicada. Universidad Simón Bolivar, Venezuela.

Pujol, L .\& Vivas, E. (1997). Prevalencia de descmpeño en lectura en niños venezolanos: dificultades según tipo de texto. Lectura y Vida. Revista Latinoamericana de Lectura. 18 (1), 17-26.

Pumfrey, P. (1978). Reading mesurement and evaluation: some current concerns and promising developments. En J. Chapman \& P. Czerniewska (Eds.). Reading from process to practice (pp. 3-5). Londres: Henley Routledge \& Kegan Paul in Association with the Open University.

Rutter, M; Tizard, J. y Whitmore, K.(1970) Educational bealth and behaviour: Londres: Logman.

Silva, P; McGee, R. y Williams, S. (1985). Some characteristics of 9 years old boys with general reading backwardess or specific reading retardation. Journal Child Psychology Psychiatric, 26 (3), 407-421.

Valencia, S. \& Pearson, D. (1987) Reading assessment: Time for change. The Reading Teacher, 20 (2), 726-731. 